\title{
Pharmacovigilance systems in resource- limited settings: an evaluative case study of Sierra Leone
}

\author{
Onome T. Abiri ${ }^{1,3^{*}}$ and Wiltshire C. N. Johnson ${ }^{2,3}$
}

\begin{abstract}
Few low and middle-income countries (LMIC) have fully operational pharmacovigilance structures, systems and legal framework to collect and collate safety data and evaluate the risks and benefits by active and passive approaches. However, in a LMIC such as Sierra Leone, the capacity to manage the risks by taking appropriate preventative actions to help inform therapeutic decisions, promote rational use of medicines, guide risk management and communications is gradually growing but yet to be fully optimized.

This study sought to assess the current status of pharmacovigilance in Sierra Leone since it became the 87th member of the World Health Organisation International Drug Monitoring Programme. This study evaluated the pharmacovigilance system in Sierra Leone through a comprehensive and system-based approach that covered the national medicines regulatory authority, health facilities and public health programmes. A descriptive cross-sectional study design was employed. Using a convenience sampling method, 14 respondents from the national medicines regulatory authority, six health facilities and six public health programmes were interviewed.

Data were collected using a validated metric instrument: Indicator-Based Pharmacovigilance Assessment Tool. A scoring system was used for the quantification of assessment results with a score greater than $60 \%$ indicating that an organization has structural and policy frameworks to collect and collate safety data in a national database and evaluate the risks and benefits by both active and passive approaches.

The study findings showed that the national medicines regulatory authority scored $79 \%$ and thus met the standard requirements of pharmacovigilance. On the other hand, the health facilities and public health programmes scored less than $60 \%$ indicating the need to fully operationalise pharmacovigilance frameworks at these levels. The study further demonstrated that the national medicine regulatory authority which hosts the national pharmacovigilance centre had functional pharmacovigilance structures and processes with potential to providing leadership in the implementation of pharmacovigilance in Sierra Leone.
\end{abstract}

Keywords: Pharmacovigilance systems, Indicators, Public health programmes, Health facilities

\section{Introduction}

The definition and scope of pharmacovigilance (PV) has evolved over the years to accommodate systems approach for improving the safe use of medicines. The World Health Organisation (WHO) has defined pharmacovigilance as the science and activities relating to the detection, assessment, understanding and prevention of

\footnotetext{
* Correspondence: berylonome@gmail.com

'Department of Pharmacology, Faculty of Basic Medical Sciences, College of Medicine and Allied Health Sciences, University of Sierra Leone, Freetown, Sierra Leone

${ }^{3}$ Pharmacy Board of Sierra Leone, Freetown, Sierra Leone

Full list of author information is available at the end of the article
}

adverse effects or any other medicine-related problems [1]. This definition covers all medicines including allopathic, complementary and alternative, biotherapeutics and vaccines.

A comprehensive PV system involves more than just risk identification and data collection. It also takes into consideration risk evaluation, minimisation and communication. Such systems protect the public from medicinerelated harms through efficient and timely detection, reporting, assessment, communication and prevention of medicine-related adverse events through people and

(c) The Author(s). 2019 Open Access This article is distributed under the terms of the Creative Commons Attribution 4.0 International License (http://creativecommons.org/licenses/by/4.0/), which permits unrestricted use, distribution, and 
structures that have the authority to take appropriate action [2].

Some local and international institutions are playing key roles in strengthening PV to guarantee medicines safety in resource-limited settings through funding mechanisms such as the US President's Emergency Plan for AIDS Relief (PEPFAR), Global Fund (GF) to fight AIDS, Tuberculosis and Malaria, the US President's Malaria Initiative (PMI) and Global Alliance for Vaccines and Immunisation (GAVI). Several technical agencies, like the WHO and its PV collaborating centres in Uppsala, Rabat, Lareb, Accra and India and the United States Pharmacopeia/Promoting the Quality of Medicines (USP/PQM) programme are also providing financial and capacity building support to build PV systems in settings were resources are scarce [2].

Funding support and initiatives by these international organisations in resource-limited countries such as Sierra Leone have resulted in increased access to medicines and vaccines for the management of public health diseases. The number of artemisinin-based combination therapy (ACT) courses procured for African countries for treatment of malaria increased from 11.2 million in 2005 to 158 million in 2009. Similarly, about four million people had access to antiretroviral therapy in sub-Saharan Africa (SSA) in 2009, compared to only 50,000 in 2002 [3]. The Global Fund alone has committed US\$ 21.9 billion with $37 \%$ of funding allocated for health commodities [4].

With increased access to newly introduced essential medicines and vaccines such as novel Ebola vaccine that would be introduced and deployed in Sierra Leone for the first time on a large scale, there is a greater need to monitor and promote their safety and effectiveness through an effective PV system. Despite extensive studies and usage of many medicines and vaccines in developed countries, their safety profiles may not be the same in other settings, given that the pattern, severity and incidence of adverse drug reaction (ADRs) may vary because of genetic and local environmental factors.

It is therefore critical that medicines continue to be monitored for safety and effectiveness as soon as they are introduced into the market under real-life conditions. For some medicines, problems will only occur in real-world situations following prolonged use, usage in specific sub-populations or in patients with various co-morbidities that are not endemic in developed countries where clinical studies were conducted and/or the products have been used [5].

The Pharmacy Board of Sierra Leone (PBSL) which is the National Medicines Regulatory Authority (NMRA), also houses the National Pharmacovigilance Centre in Sierra Leone. As a full member of the WHO International Drug Monitoring Programme, the PBSL promotes and supports PV in health facilities, public health programmes, universities and research institutions through the establishment of effective medicines monitoring system.

An effective PV system protects the public through efficient and prompt recognition, collection, recording and evaluation of adverse events and by communicating benefits and risks to corroborate therapeutic decision-making at different strata of the health care system. Few low and middle-income countries have fully operational PV structures, systems and legal frameworks to collect and collate safety data and evaluate the risks and benefits by both passive and active approaches. However, in countries such as Sierra Leone, the capacity to manage the risks by taking appropriate preventive actions to help inform therapeutic decisions, promote rational use of medicines and guide risk management and communication is gradually growing but yet to be fully optimised.

Of 46 SSA countries studied by the Strengthening Pharmacovigilance Systems (SPS) programme, 87\% did not have a functional PV system, 59\% did not have a national policy related to medicine safety, $70 \%$ lacked legislation to monitor adverse events, $26 \%$ did not have a national PV center, and 61\% lacked a medicine safety advisory committee. Furthermore, $74 \%$ was reported to have spontaneous adverse event reporting systems, while less than $50 \%$ monitored product quality, medication errors, or treatment failures [6]. A similar study conducted in five Asian countries namely Bangladesh, Cambodia, Nepal, Thailand and the Philippines indicated that amongst the 86 health facilities evaluated, less than 50\% of them had a PV center or unit, or designated staff for PV-related activities within their facilities. Fifteen percent had Drug and Therapeutics Committee (DTCs) and 38\% had Drug Information Services (DIS), while for the 19 Public Health Programmes (PHPs) assessed, 26\% were reported to have dedicated funds available, 53\% had Standard Operating Procedures (SOPs) and 58\% had guidelines in place that addressed elements of PV [7].

Since no PV assessment has been done in Sierra Leone from the time it became the 87th member of the WHO International Drug Monitoring programme in 2008, this study therefore aimed to evaluate the current status of PV in Sierra Leone through a comprehensive and system-based approach that covered the PBSL, healthcare facilities and PHPs.

\section{Methods}

\section{Study setting}

Sierra Leone is a country in West Africa that is bordered by Guinea to the northeast, Liberia to the southeast, and the Atlantic Ocean to the southwest. The country covers a total area of $71,740 \mathrm{~km}^{2}(27,699$ sq. mile) and with an estimated population of 7 million [8]. Sierra Leone is divided into five administrative regions: the Northern 
Region, North-Western Region, Eastern Region, Southern Region and the Western Area; which are subdivided into 16 districts. The national public health system of Sierra Leone consists of 986 peripheral health units, 53 secondary health facilities and a teaching hospital complex that consist of six tertiary referral teaching hospitals (8). The ratios of physician and pharmacist to patient population is $2: 50,000$ and $1: 50,000$ respectively $[9,10]$.

\section{Study design and sampling}

This was a descriptive cross-sectional study that was conducted in Sierra Leone from April to September 2016. Fourteen respondents recruited by convenience sampling method from the PBSL, six health facilities and six PHPs were interviewed. The study participants consisted of two pharmacists from PBSL, three pharmacists and three medical doctors from the health facilities and six pharmacists from the PHPs. Study participants were recruited from the following institutions:

- The PBSL

- Six health facilities

oThree teaching hospitals located in the capital city, Freetown:

- Connaught Teaching Hospital (CTH)

- Ola During Children Hospital (ODCH)

- Princess Christian Maternity Hospital (PCMH)

oThree regional referral hospitals located in the southern, eastern and northern regions respectively):

- Bo government hospital (BoGH)

- Kenema government hospital (KeGH)

- Makeni government hospital (MaGH)

- Six Public Health Programmes (PHPs):

- The National Malaria Control Programme (NMCP) oNational Tuberculosis and Leprosy Control Programme (NLTCP)

oThe HIV/AIDS Control Programme (HIV/AIDS) oThe Reproductive and Child Health Programme ( $\mathrm{RCH})$

oThe Expanded Programme on Immunisation (EPI) -The Neglected Tropical Disease Control Programme (NTDCP)

\section{Data collection \\ Data collection tool}

The Indicator-based Pharmacovigilance Assessment Tool (IPAT) (2) was used for data collection. The tool was designed and validated by the Management Sciences for Health, a US-based not-for--profit organization through its strengthening of pharmaceutical systems programme specifically for the assessment of pharmacovigilance systems in developing countries.

IPAT has 43 indicators including 26 core and 17 supplementary indicators. These indicators cover five pharmacovigilance medicine safety monitoring components. The purpose of the tool is to enable the conduct of pharmacovigilance assessment through a series of questions focusing on structures, processes and impact of pharmacovigilance systems. The five areas of medicine safety monitoring covered are:

1. Policy, law and regulation (4 indicators, 1.1-1.4)

2. Systems, structures and stakeholder coordination (15 indicators, 2.1-2.15)

3. Signal generation and data management (6 indicators, 3.1-3.6)

4. Risk assessment and evaluation (8 indicators, 4.1-4.8)

5. Risk management and communication (10 indicators, 5.1-5.10)

\section{Data collection level}

The first area (policy, law and regulation) represents data collected at the national level such as the NMRA. The other four areas are important for data collection for hospitals and public health programmes.

In this study, the indicators were classified according to their relevance to health system levels and where they could be collected:

- 39 indicators for NMRAs (1.1-1.4, 2.1-2.15, 3.1-3.6, 4.1-4.5, 5.1-5.7 and 5.9-5.10)

- 21 indicators for hospitals $(2.1-2.5,2.8-2.11,2.13$, 3.3-3.6, 4.1, 4.3-4.5, 5.1 and 5.3-5.4)

- 25 indicators for PHPs $(1.1,2.1-2.2,2.4-2.5,2.8-2.9$, $2.13,3.3-3.6,4.1-4.8,5.1-5.3,5.7$ and 5.9$)$

\section{Data collection process}

Prior to data collection, the participants were requested to take part in the study through face to face contacts, emails and telephone calls. The respondents provided information with respect to the indicators on the IPAT tool administered to them through face-face interviews from April to June 2016. Relevant documents on PV were also collected from the respondents and reviewed by the investigators. These documents served as evidence in support of the interviews. For the NMRA, documents reviewed were National Medicines Policy 2012, Pharmacy and Drugs Act 2001, revised Pharmacy and Drugs Act 2012, 2015 annual report, 2015 regulatory and medicines information bulletins, pharmacovigilance guidelines. Documents reviewed from the health facilities included 2015 annual reports, job descriptions and SOPs of PV focal persons and treatment guidelines. The PHPs documents reviewed covered treatment guidelines, policies, SOPs and 2015 annual reports. 


\section{Data analysis}

Analysis of data was quantitative and qualitative and Microsoft Excel (Microsoft Corporation, Redmond, WA, USA) was used to compute the scores. With respect to scoring of the indicators, a core indicator recorded as 'Yes' was allocated a score of 2; a supplementary indicator recorded as 'Yes' was allocated a score of 1; any core or supplementary indicator recorded as 'No' was given a score of 0 . These numerical values assigned are in line with the IPAT tool scoring system. Recommended thresholds were indicated by the IPAT manual for the quantitative indicators, i.e. indicators with numbers and percentages $(2.13,4.4,5.3,5.4,5.5,5.6,5.7$ and 5.10), resulting in a total score of 52 for core indicators and 17 for the supplementary indicators. The score attained by the indicators was divided by the total score of all the indicators and multiplied by 100 . If this value is greater than $60 \%$ for each component of health system level, the component is said to meet the standard PV requirements for that health system level $(2,6)$. Results were presented as tables and were used to compare performance of indicators with the same component.

\section{Ethical considerations}

Authorisation to conduct the study was obtained from the relevant institutions where the study was done, that is, the Pharmacy Board of Sierra Leone, the PHPs and hospitals. The participants gave their consent to take part in the study before they were interviewed. Data were processed anonymously to ensure confidentiality.

\section{Results}

Fourteen respondents from 13 institutions including two respondents from PBSL, six from health facilities and six from PHPs were interviewed. The sample size consisted of 11 pharmacists and 3 medical doctors.

\section{Pharmacovigilance performance of PBSL}

Thirty-nine indicators (23 core and 16 supplementary) and (19 structure, 11 process and 9 outcome) were employed to assess the PBSL for a maximum score of 62 (Additional file 1: Table S1. Result for the Pharmacy Board of Sierra Leone).

Pharmacy Board of Sierra Leone got an overall score of 79\% (49/62). The performance by pharmacovigilance (PV) area is as follows (Table 1):

The findings of the study revealed that the required policy statements for policy, law, and regulation for PV in Sierra Leone was in place but no specific legislation for PV was available. The National Medicines Policy (NMP) recognized the need for PV and mandated PBSL with the responsibility for pharmacovigilance and clinical trial regulation [11].
Table 1 Pharmacovigilance performance of PBSL

\begin{tabular}{lll}
\hline PV component & Score (\%) & Target outcome \\
\hline $\begin{array}{l}\text { Policy, law and regulation } \\
\text { Systems, structures and }\end{array}$ & $33(2 / 6)$ & Not achieved \\
stakeholders coordination & $96(25 / 26)$ & Achieved \\
Signal generation and management & $100(12 / 12)$ & Achieved \\
Risk assessment and evaluation & $43(3 / 7)$ & Not achieved \\
Risk management and communication & $64(7 / 11)$ & Achieved \\
Overall score & $79(49 / 62)$ & Achieved \\
\hline
\end{tabular}

However, specific legal provision for the enforcement of PV is currently not available in Sierra Leone as the current Sierra Leone Pharmacy and Drugs Act 2001 does not include pharmacovigilance. The Pharmacy and Drugs Act also did not place responsibility on marketing authorisation holders (MAHs) for product stewardships with respect to mandatory reporting of all serious adverse events (SAEs) or suspected unexpected serious adverse reaction (SUSARs) to the NMRA and conduct of post-marketing surveillance activities (Table 2).

Consequently, the Pharmacy and Drugs Act has been revised by the NMRA and presented for ratification by the parliament. PBSL has also developed guidelines to implement a system of Qualified Person for Pharmacovigilance (QPPV) that will mandate and ensure that MAHs have functional pharmacovigilance systems in place so that they can assume responsibility and liability for their products in the market and to ensure that appropriate actions are taken when necessary [12].

\section{Pharmacovigilance performance of health facilities}

Twenty-two indicators (15 core and 7 supplementary) and (10 structure, 8 process and 4 outcome) were employed to assess the health facilities for a maximum score of 37 (Additional file 1: Table S2. Result for health facilities).

The overall scores for the health facilities were (Table 3):

\section{Pharmacovigilance performance of public health programmes (PHPs)}

Twenty-five indicators (16 core and 9 supplementary) and (8 structure, 12 process and 5 outcome) were employed to assess the PHPs for a maximum score of

Table 2 Policy, law and regulation

\begin{tabular}{ll}
\hline Policy, law and regulation & Document Availability \\
\hline PV policy & Available \\
PV legislation & Not available \\
Legal provision for MAHs to report ADRs & Not available \\
Legal provision for MAHs to conduct & Not available \\
post-marketing surveillance activities & \\
\hline
\end{tabular}


Table 3 Pharmacovigilance performance health facilities

\begin{tabular}{lll}
\hline Health facilities & Score (\%) & Target outcome \\
\hline Connaught Teaching Hospital & $51(19 / 37)$ & Not achieved \\
Princess Christian Maternity Hospital & $51(19 / 37)$ & Not achieved \\
Ola During Children Hospital & $22(8 / 37)$ & Not achieved \\
Bo Government Hospital & $54(20 / 37)$ & Not achieved \\
Kenema Government Hospital & $54(20 / 37)$ & Not achieved \\
Makeni Government Hospital & $54(20 / 37)$ & Not achieved \\
\hline
\end{tabular}

41 (Additional file 1: Table S3. Results for the public health programmes).

The overall scores for the PHPs were (Table 4):

Of the PHPs assessed, only 2 of 6 (33\%) i.e. neglected diseases and immunisation programmes reported having a policy document containing essential statements on PV.

\section{Discussion}

The national PV centre of the NMRA, Pharmacy Board of Sierra Leone scored $79 \%$ and met the standard requirements of PV by scoring greater than $60 \%$ while none of the health facilities and PHPs satisfied these criteria.

The lack of relevant PV legislations and regulations in Sierra Leone reflects fundamental limitation for enforcing medicine safety monitoring. This omission limits the capacity PBSL to mandate post-marketing safety commitments of the MAHs. Existence of a legislation, guidelines and policies having relevant statements on PV shows that a country has demonstrated high-level commitment to improve medicine safety and hence provide direction to enhance the system. The existence of legislations and regulations also support a strong legal foundation that ensures conformity by relevant stakeholders.

A similar study done in sub-Saharan Africa (SSA) that included 46 countries, showed that 19 countries (41\%) have a national policy related to PV; 14 countries (30\%) provided a legal mandate to monitor medicine-related adverse events. Only 13 countries (28\%) have legal

Table 4 Pharmacovigilance performance of public health programmes

\begin{tabular}{lll}
\hline Public health programmes & Score (\%) & Target outcome \\
\hline National Malaria Control Programme & $54(22 / 41)$ & Not achieved \\
Neglected Tropical Diseases & $51(21 / 41)$ & Not achieved \\
Control Programme & & \\
Expanded Programme on Immunisation & $44(18 / 41)$ & Not achieved \\
HIV/AIDS Control Programme & $37(15 / 41)$ & Not achieved \\
$\begin{array}{l}\text { National Leprosy and Tuberculosis } \\
\text { Control Programme }\end{array}$ & $39(16 / 41)$ & Not achieved \\
Reproductive and Child Health & $34(14 / 41)$ & Not achieved \\
Programme & & \\
\hline
\end{tabular}

provisions that require MAHs to report all serious ADRs to the NMRA and eight countries (17\%) require MAHs to conduct post-marketing surveillance activities [6].

Systems, structures, and stakeholder coordination have grown over the years with respect to PV in Sierra Leone. Sierra Leone has established basic structures for conducting PV activities, including a PV centre with clear mandate, defined roles and responsibilities, formal organizational structure, designated persons for pharmacovigilance, functional information and communication technology infrastructure, a national PV guideline, a medicine safety advisory committee and collaboration with the Uppsala Monitoring Centre (UMC) which is the World Health Organisation (WHO) Collaborating Center for International Drug Monitoring in Uppsala, Sweden. Through this collaboration, Sierra Leone became the 87th member of the WHO programme in 2008.

However, across the health facilities and public health programmes, lack of a dedicated budget for PV, pharmacovigilance training for health care workers, standard operating procedures (SOPs) and guidelines for PV-related activities, drug information services (DIS) and drug and therapeutics committees (DTCs) performing PV-related activities was reported. Kabore and colleagues also published results compatible to this study [13].

The development of sustainable PV systems and their optimal functioning are critical for effective medicines safety monitoring. Some of the factors required for a sustainable PV system include political will, financial support from government and partners; and also, strong collaborations and cooperation of health care professionals and health institutions.

Structures, systems and roles provide a foundational basis for organised and systematic operationalisation of PV activities. This will enable effective and efficient use of staff, skills, and tools. The implications of the lack of PV guidelines in health facilities and PHPs can lead to the inability to map and streamline stakeholders' contributions to PV. This paucity has also contributed to the poor functioning of PV structures and systems within the health facilities and PHPs in relation to implementing and advancing PV activities. The development and implementation of comprehensive guidelines and SOPs will serve as a basis for structured and coordinated PV activities and optimum performance by various stakeholders. The lack of dedicated PV budget, procedures and training in pharmacovigilance for healthcare professionals indicates the inability and lack of capacity to consistently address PV issues. Where there are no proper structures, systems, and clear roles and responsibilities, there will be no fundamental basis for organised and systematic operationalisation of pharmacovigilance activities.

For signal generation and data management, the national PV centre has ADR reporting forms for consumer 
reporting, mass medicines administration campaigns and health care professionals. The spontaneous reporting system covers the full scope of PV, including product quality, medication errors, and treatment failures that can be reported by using the current ADR form. The central database of the national PV centre contains data from various sources, such as reports from clinical trials, adverse event following immunisation (AEFI) from the EPI/Child Health Programme, and ADRs from PHPs such the Malaria Control programme, the Tuberculosis and Leprosy Control Programme, Neglected Tropical Disease Control Programme and health facilities. Serious adverse event (SAE) reports from clinical trials are sent to the national PV centre, hence, there is an opportunity to draw from the inherent advantage of linking pre-marketing phases I, II and III and post-marketing safety data. Such coordination of PV data from various sources will enhance the effective synthesis, interpretation, and use of safety information. However, low reporting rates from health facilities and PHPs to the PV centre can be a challenge for signal generation.

Signal detection through reporting of suspected adverse events is the first step in the PV process, followed by signal evaluation and risk management [14]. Generation of signals depends on well sensitised healthcare professionals who report suspected adverse events. With this poor reporting rate observed in this study of suspected adverse events such as treatment failure and poor product quality, one can conclude that the national PV centre has few reports in its database, which will make detection and generation of signals to address safety issues challenging.

In Sierra Leone, factors such as lack of awareness of healthcare professionals and resources to promote pharmacovigilance contribute to poor ADR reporting rates. Low reporting rates of ADRs have implications for the ability to generate and evaluate signals of public health significance. When such risk assessment efforts are not undertaken, opportunities to learn about the safety and effectiveness of medicines during real-life use are lost. Subsequently, opportunities to use such new knowledge to inform treatment decisions by clinicians and pharmacists are also not utilised.

In relation to risk assessment and evaluation, the study revealed that no medicine utilisation reviews, studies quantifying incidence of medication errors and active surveillance studies have been conducted over the past years. The periodic review of the number and types of medicine-related adverse events through passive surveillance reporting as well as evaluation of significant safety issues through active surveillance are fundamental attributes of any comprehensive pharmacovigilance system. With respect to the number of ADRs reported in the previous year, IPAT recommends the use of thresholds to determine whether the number of reported adverse events meets that expected for a minimally functional PV system. For example, using a threshold of 100 reports per million populations per year, Sierra Leone will be expected to generate about 700 reports per year, and a health facility with about 50,000 people in its catchments area will be expected to generate a minimum of 5 reports per year to meet the threshold. The number of ADR reports received in Sierra Leone for the year under review was 1084 according to the PBSL 2015 annual report [15].

However, 95\% of these reports were obtained from mass medicine distribution campaigns with artesunateamodiaquine and albendazole-ivermectin, which suggested that the reporting rate might drop in the coming years without such initiatives. A study conducted by the strengthening pharmaceutical systems programme revealed that reporting rate of adverse event was minimal in the 46 SSA countries that were surveyed; only two of the countries surveyed collected more than 100 reports per million populations in 2010 (i.e. Burkina Faso and Namibia), and most countries generated less than 20 reports per million populations per year [6].

Without collection, reporting and analysis of adverse events, signals of public health importance will be missed and opportunities to learn about the safety and effectiveness of medicines during real-life use will also be lost. This finding has consequences for the ability to generate signals and evaluate safety issues of public health importance. Acknowledging that PV is still relatively new to Sierra Leone and that the PV centre became a member of the WHO programme in 2008, considerable effort and time are required to raise awareness among health care professionals and consumers on the significance of reporting adverse events.

From the risk management and communication perspective, this study showed that the PV centre requires MAHs to have risk mitigation plans (RMP) for high-risk medicines so as to prevent and manage ADRs by averting serious known risks. In Sierra Leone, between 2015 and 2016, MAHs of products such as Tivicay (Dolutegravir) for HIV/AIDS, Orasure and Congenix Ebola diagnostics were required to submit risk management plan (RMP) to the NMRA and are been monitored on a quarterly basis to ensure compliance [15]. Requesting safety information, responding to safety information, using bulletins to publish and communicate safety information to health care workers and the community still require considerable work so as to bolster risk management and communication across all health institutions. Risk management and communication is a crucial aspect of PV with high impact in preventing harm from medicines use. The essential medicines list contains products such as the fluoroquinolones and antiretrovirals that require risk management, 
but no strategies are in place to ensure safe use of these products in health facilities and PHPs. These facilities do not have guidelines for managing high-risk medicines. This situation can immensely affect the quality of patient care. The application of medicines utilisation reviews, risk management, and risk communication can enable PV to contribute to the improvement of health outcomes.

This study has some limitations. Firstly, a small number of study participants recruited through convenience sampling was employed for this study. Other key PV stakeholders such as health professional bodies such as the Sierra Leone Medical and Dental Council, Nurses Board. and private sectors were not included. This could affect the generalisability of the results. Secondly, the study relied on respondents' assertions unless in cases where documentations were available as a source of verification. Nevertheless, the findings of the study have potential for providing platform for future research agenda on PV implementation in Sierra Leone and similar settings.

\section{Conclusion}

The study demonstrates that PBSL which hosts the national PV centre, had the basic PV structures and processes in place and showed potential to providing leadership in implementation of PV in Sierra Leone. However, the study uncovered some gaps, mainly related to the lack of pharmacovigilance-specific legislation. The Pharmacy Board of Sierra Leone lacks the regulatory framework mandated by law and capacity for enforcement in order to ensure that industries assume responsibilities and liabilities for their products in the market and take appropriate actions when necessary.

Health facilities and PHPs have PV systems and structures that are currently weak and the ability to generate signals, evaluate them and use the information for risk management and communication is limited. Incorporation of both active and passive approaches with careful strategic planning can improve the impact of PV and consequently, improve patient safety. A great challenge and opportunity exist to improve the systems and capacities required to assure patient safety and to improve health outcomes in Sierra Leone.

Therefore, PV legislation should be in place to adequately address medicines safety monitoring nationwide and this should be backed with sufficient and dedicated budget. Active surveillance activities such as registries, sentinel sites and cohort event monitoring should be incorporated into the national PV system through close collaborations with research institutions, universities and PHPs so as to increase ADR reporting and aid signal detection. The National PV Centre of PBSL should collaborate with health professional regulatory bodies such as the Sierra Leone Medical and Dental Council, the
Pharmaceutical Society of Sierra Leone, the Nurses Board and the universities teaching health-related discipline to ensure locally relevant PV topics are included in pre- and in-service training programmes.

\section{Additional file}

Additional file 1: Table S1. Result for the Pharmacy Board of Sierra Leone. Table S2. Result for health facilities. Table S3. Result for the public health programmes. (DOCX $40 \mathrm{~kb}$ )

\section{Abbreviations}

ADR: Adverse Drug Reaction; IPAT: Indicator-based Pharmacovigilance Tool; MAHs: Marketing Authorisation Holders; NMRA: National Medicine Regulatory Authority; PBSL: Pharmacy Board of Sierra Leone; PHPs: Public Health Programmes, PVPharmacovigilance; QPPV: Qualified Person for Pharmacovigilance; WHO: World Health Organisation

\section{Acknowledgements \\ The authors wish to acknowledge, staff of the pharmacovigilance department of the Pharmacy Board of Sierra Leone, the managers and pharmacists of the following programmes; national malaria control, the national leprosy and tuberculosis control, HIV/AIDS control, expanded programme on immunisation, neglected tropical diseases and reproductive and child health and also the medical superintendents and hospital pharmacists of Connaught hospital, Ola During children hospital, Princess Christian maternity hospital, Bo government hospital, Kenema government hospital and Makeni government hospital for the information provided for this study. The authors also thank the Management Sciences for Health for giving us permission to use their indicator-based phar- macovigilance assessment tool.}

\section{Funding}

This study was self-funded as part of a fellowship examination of the West African Postgraduate College of Pharmacists (WAPCP).

\section{Availability of data and materials}

The dataset used and analysed during the study are available from the corresponding author on request.

\section{Authors' contributions}

OTA and WCNJ contributed to the conceptualisation and the study design. Collection and analysis of data was done by OTA. OTA drafted the manuscript and all authors reviewed and agreed on the final manuscript for submission. WCNJ was the supervisor and OTA was the fellowship candidate. All authors read and approved the final manuscript.

\section{Ethics approval and consent to participate}

Authorisation to conduct the study was solicited from the relevant institutions where the study was done that is the Pharmacy Board of Sierra Leone, PHPs and health facilities. The participants gave their consent to take part in the study before they were interviewed. Data was processed anonymously to ensure confidentiality.

\section{Consent for publication}

The authors have all agreed to the publication in this journal.

Competing interests

The authors declare that they have no competing interests.

\section{Publisher's Note}

Springer Nature remains neutral with regard to jurisdictional claims in published maps and institutional affiliations.

\section{Author details}

'Department of Pharmacology, Faculty of Basic Medical Sciences, College of Medicine and Allied Health Sciences, University of Sierra Leone, Freetown, Sierra Leone. ${ }^{2}$ Department of Clinical Pharmacy and Therapeutics, Faculty of Pharmaceutical Sciences, College of Medicine and Allied Health Sciences, 
University of Sierra Leone, Freetown, Sierra Leone. ${ }^{3}$ Pharmacy Board of Sierra Leone, Freetown, Sierra Leone.

Received: 14 September 2017 Accepted: 29 March 2019

Published online: 11 June 2019

\section{References}

1. World Health Organisation [WHO]. The importance of pharmacovigilance. Geneva: World Health Organisation; 2002.

2. Strengthening Pharmaceutical Systems [SPS]. Supporting Pharmacovigilance in Developing Countries: The Systems Perspective. Submitted to the U.S. Agency for International Development by the SPS Program. Arlington, VA: Management Sciences for Health; 2009.

3. World Health Organisation [WHO] (2010). Towards universal access: scaling up priority HIV/AIDS interventions in the health sector. [online]. Available at: http://www.who.int/hiv/pub/2010progressreport/report/en/index.html [Accessed 1 September 2016].

4. Global Fund [GF]. (2011). The Global Fund to Fight AIDS, Tuberculosis and Malaria (Global Fund). Report of the Market Dynamics and Commodities Ad-Hoc Committee to 23rd Board Meeting. [online]. Available at: http://www. theglobalfund.org/documents/board/23/BM23_09MDC_ [Accessed 1 September 2016].

5. Pirmohamed M, Atuah KN, Dodoo AN, Winstanley P. Pharmacovigilance in developing countries. BMJ: Br Med J. 335(7618):462.

6. Strengthening Pharmaceutical Systems [SPS]. Safety of Medicines in Sub-Saharan Africa: Assessment of Pharmacovigilance Systems and their Performance. Arlington, VA: US Agency for international development by the strengthening pharmaceutical Systems (SPS) program; 2011.

7. System for Improved Access to Pharmaceuticals and Services [SIAPS] Comparative analysis of pharmacovigilance systems in five Asian countries. Arlington, VA: Systems for improved access to pharmaceuticals and services. Management Sciences for Health; 2013.

8. Statistics Sierra Leone [SSL] (2016). Sierra Leone Housing and Population Census. Provisional result [online]. Available at: https:/www.statistics.sl/ [Accessed 2 September 2016].

9. Government of Sierra Leone [GoSL]. The Sierra Leone Gazette (Extraordinary): Pharmacy Board of Sierra Leone. Freetown: Government of Sierra Leone; 2016.

10. Sierra Leone Medical and Dental Association [SLMDA] (2015). Sierra Leone Medical and Dental Association Annual Congress and Scientific meeting. Freetown: Sierra Leone medical and Dental Association.

11. Ministry of Health and Sanitation [MOHS]. National Medicines Policy. Freetown: Directorate of Drugs and Medical Supplies, Ministry of Health and Sanitation; 2012.

12. Pharmacy Board of Sierra Leone [PBSL] (2016). Guideline for marketing authorisation holders: requirements for qualified person for pharmacovigilance (QPPV) [online]. Available at: http://www.pharmacyboard.gov.sl/site/ Downloads/Guidelines.aspx [Accessed 1st September 2016].

13. Kabore L, Millet P, Fofana S, Berdai D, Adam C, Haramburu F. Pharmacovigilance systems in developing countries: an evaluative case study in Burkina Faso. Drug Saf. 36(5):349-58

14. Bisson G, Gross R, Miller V, Weller I, Walker A. Monitoring of long-term toxicities of HIV treatments: an international perspective. Aids. 17(17):2407-17.

15. Pharmacy Board of Sierra Leone [PBSL] (2015). Pharmacy Board of Sierra Leone Annual Report.

Ready to submit your research? Choose BMC and benefit from:

- fast, convenient online submission

- thorough peer review by experienced researchers in your field

- rapid publication on acceptance

- support for research data, including large and complex data types

- gold Open Access which fosters wider collaboration and increased citations

- maximum visibility for your research: over $100 \mathrm{M}$ website views per year

At $\mathrm{BMC}$, research is always in progress.

Learn more biomedcentral.com/submissions 